\title{
Entangled detectors nonperturbatively harvest mutual information
}

\author{
Kensuke Gallock-Yoshimura ${ }^{1, *}$ and Robert B. Mann ${ }^{1,2, \text {, }}$ \\ ${ }^{1}$ Department of Physics and Astronomy, University of Waterloo, Waterloo, Ontario, N2L 3G1, Canada \\ ${ }^{2}$ Perimeter Institute for Theoretical Physics, Waterloo, Ontario, N2L 2Y5, Canada
}

\begin{abstract}
We investigate how entangled inertial Unruh-DeWitt detectors are affected by interaction with a quantum field using a nonperturbative method. Inertial detectors in a $(3+1)$-dimensional Minkowski spacetime with instantaneous switching $(\delta$-switching) experience degradation of their initial entanglement as their coupling strength with a scalar field increases. Somewhat surprisingly, initially separable or weakly entangled detectors can extract mutual information from the vacuum. We also find that entanglement degradation is not reduced if communication via the field is possible; rather this only changes the manner in which entanglement is degraded.
\end{abstract}

\section{INTRODUCTION}

The fact that quantum fields are entangled [1, 2] even in the vacuum state has attracted increasing attention over the past decade. Originally, Valentini $[\underline{3}$ and subsequently Reznik et al. [4, 5] showed that particle detectors can become entangled by extracting correlations in the field, even without directly exchanging quanta. This kind of entanglement extraction from the field is called entanglement harvesting, and has been examined extensively [6 20]. The general approach has been to consider initially separable Unruh-DeWitt (UDW) detectors [21, 22 interacting with a scalar field for a finite time. As long as no angular momentum is exchanged, this is a good approximation to the light-matter interaction [23 25$]$.

While entanglement harvesting is about extracting entanglement from the field, there is a phenomenon called entanglement degradation, in which the amount of a prepared entanglement is reduced due to the field. Typically, the Unruh and Hawking effects have been of primary interest [26-34]: maximally entangled field modes from an inertial observer's viewpoint will be less entangled from an accelerating observer's point of view because of the noisy Unruh effect. In addition, it is well known that entanglement of bosonic fields vanishes in the large acceleration limit, while that of fermionic fields survives [27, 32, 33, 35. In the context of the UDW detectors, initially entangled, noninertial detectors lose some (or all) of the correlation after interacting with the field $[29,36,37$.

Our interest is whether or not an initial entanglement between two detectors is always degraded. While initially entangled detectors experience entanglement degradation, it is conceivable that they could also gain entanglement from the vacuum according to the entanglement harvesting protocol. Moreover, communication between the detectors enhances entanglement in the harvesting scenario, but it is not clear how communication plays a role in entanglement degradation. In fact, it has been shown [36] that entanglement can be revived for a while even after detectors are completely disentangled. It is

\footnotetext{
* kgallock@uwaterloo.ca

$\dagger \frac{\text { rbmann@uwaterloo.ca }}{\text { rba }}$
}

therefore of interest to see in what circumstances initially entangled detectors gain or lose entanglement, or more generally gain or lose correlation.

To this end, we nonperturbatively analyze two inertial UDW detectors coupled to a scalar field in $(3+1)$ dimensional Minkowski spacetime, while paying attention to their communication channels. We will make use of a nonperturbative analysis [11] in which the UDW detectors are assumed to switch on and off instantaneously, modeled by a Dirac $\delta$-function. Such a method enables us to obtain a density matrix of detectors that clearly shows how communication and the initial state contribute to entanglement degradation.

Our main findings are the following. (a) Detectors experience entanglement degradation as their coupling with the field gets stronger if only one of or both of the detectors interact with the field. (b) Although mutual information (classical and quantum correlations) between the detectors exhibits similar behavior to entanglement, weakly entangled detectors can actually gain mutual information from the vacuum, even without communication. (c) Communication does not enhance either entanglement or mutual information. However distinct initial entangled states with the same amount of entanglement show different degradation properties, which purely originate from communication.

Our paper is organized as follows. In Sec. IIA we review the UDW detectors with the "delta switching" (i.e., interacting instantaneously). Then we derive the density matrix of the detectors after the interaction in IIB. To be more specific, it is a density matrix of two initially entangled, inertial detectors in an $(n+1)$-dimensional Minkowski spacetime. We then further assume $n=3$ and that the detectors' shapes are both specified by a Gaussian smearing function. We give measures of correlations, concurrence, and mutual information in Sec. IIC Results are shown in Sec. III. In particular, we consider two cases: only one detector interacts with the field (III A) and both detectors interact (IIIB) after the entangled detectors are prepared. Finally we conclude in Sec. IV] Throughout the paper, we use natural units $\hbar=c=1$, and denote spacetime points by $\mathrm{x}=(t, \boldsymbol{x})$. 


\section{UDW DETECTORS WITH DELTA SWITCHING}

\section{A. Time-evolution operator}

Consider two UDW detectors A and B interacting with a local quantum field $\hat{\phi}(\mathrm{x})$. The interaction Hamiltonian of a linearly coupled detector- $j \in\{\mathrm{A}, \mathrm{B}\}$ is given by

$$
\hat{H}_{j}^{\tau_{j}}\left(\tau_{j}\right)=\lambda_{j} \chi_{j}\left(\tau_{j}\right) \hat{\mu}_{j}\left(\tau_{j}\right) \otimes \int \mathrm{d}^{n} x F_{j}\left(\boldsymbol{x}-\boldsymbol{x}_{j}\right) \hat{\phi}\left(\mathrm{x}_{j}\left(\tau_{j}\right)\right),
$$

where $\tau_{j}, \lambda_{j}, \chi_{j}\left(\tau_{j}\right)$, and $F_{j}\left(\boldsymbol{x}-\boldsymbol{x}_{j}\right)$ are the proper time, the coupling constant, the switching function, and the smearing function of detector- $j$, respectively. The superscript $\tau_{j}$ indicates that it is a generator of timetranslations with respect to $\tau_{j}$. The switching function $\chi_{j}\left(\tau_{j}\right)$ determines the time-dependence of coupling, and the smearing function $F_{j}\left(\boldsymbol{x}-\boldsymbol{x}_{j}\right)$ represents the size and the shape of a detector whose center of mass is located at $\boldsymbol{x}_{j}$. The operator $\hat{\mu}_{j}\left(\tau_{j}\right)$ is the monopole moment given by

$$
\hat{\mu}_{j}\left(\tau_{j}\right)=\left|e_{j}\right\rangle\left\langle g_{j}\left|e^{i \Omega_{j} \tau_{j}}+\right| g_{j}\right\rangle\left\langle e_{j}\right| e^{-i \Omega_{j} \tau_{j}},
$$

where $\left|g_{j}\right\rangle,\left|e_{j}\right\rangle$ are the respective ground and excited states of detector- $j$, and $\Omega_{j}$ is the energy gap between these two states.

Let us employ the switching function defined by [11]

$$
\chi_{j}\left(\tau_{j}\right)=\eta_{j} \delta\left(\tau_{j}-\tau_{j, 0}\right),
$$

which we refer to as delta switching, where $\tau_{j, 0}$ is the proper time when detector- $j$ is switched, and $\eta_{j}$ is a constant, which has units of time, so that

$$
\int_{-\infty}^{\infty} \chi_{j}\left(\tau_{j}\right) \mathrm{d} \tau_{j}=\eta_{j}
$$

is constant.

By making use of delta switching, we can analyze the properties of detectors nonperturbatively. Let us introduce a coordinate system $(t, \boldsymbol{x})$ that specifies the positions of both detectors. In our analysis, such a coordinate system will be Cartesian coordinates in Minkowski spacetime. Since the time component $t$ can specify time for both detectors, the proper times $\tau_{j}$ can be written in terms of $t$, i.e., $\tau_{j}(t)$. By using this common time $t$, the time-evolution operator $\hat{U}_{\mathrm{I}}$ in the interaction picture can be written as follows.

$$
\hat{U}_{\mathrm{I}}=\mathcal{T}_{t} \exp \left[-\mathrm{i} \int_{\mathbb{R}} \mathrm{d} t \hat{H}_{\mathrm{I}}^{t}(t)\right],
$$

where $\mathcal{T}_{t}$ is time-ordering symbol with respect to the common time $t$, and the Hamiltonian $\hat{H}_{\mathrm{I}}^{t}(t)$ is [38, 39]

$$
\hat{H}_{\mathrm{I}}^{t}(t)=\frac{\mathrm{d} \tau_{\mathrm{A}}}{\mathrm{d} t} \hat{H}_{\mathrm{A}}^{\tau_{\mathrm{A}}}\left(\tau_{\mathrm{A}}(t)\right)+\frac{\mathrm{d} \tau_{\mathrm{B}}}{\mathrm{d} t} \hat{H}_{\mathrm{B}}^{\tau_{\mathrm{B}}}\left(\tau_{\mathrm{B}}(t)\right) .
$$

Assuming that detector A switches earlier than B, namely, $t\left(\tau_{\mathrm{A}, 0}\right) \leq t\left(\tau_{\mathrm{B}, 0}\right)$, the delta switching enables us to write the time-evolution operator $\hat{U}_{\mathrm{I}}$ as $[11$

$$
\hat{U}_{\mathrm{I}}=\exp \left[\hat{\mu}_{\mathrm{B}}\left(\tau_{\mathrm{B}, 0}\right) \otimes \hat{Y}_{\mathrm{B}}\left(\tau_{\mathrm{B}, 0}\right)\right] \exp \left[\hat{\mu}_{\mathrm{A}}\left(\tau_{\mathrm{A}, 0}\right) \otimes \hat{Y}_{\mathrm{A}}\left(\tau_{\mathrm{A}, 0}\right)\right],
$$

where

$$
\hat{Y}_{j}\left(\tau_{j, 0}\right):=-\left.\mathrm{i} \lambda_{j} \eta_{j} \frac{\mathrm{d} \tau_{j}}{\mathrm{~d} t}\right|_{\tau_{j, 0}} \int \mathrm{d}^{n} x F_{j}\left(\boldsymbol{x}-\boldsymbol{x}_{j}\right) \hat{\phi}\left(\mathrm{x}_{j}\left(\tau_{j, 0}\right)\right)
$$

The operator $\hat{Y}_{j}$ is essentially a smeared field operator at the switching-time $t\left(\tau_{j, 0}\right)$. We finally define a commutator $\Theta$ and an anti-commutator $\omega$ as

$$
\begin{aligned}
\Theta & :=-\mathrm{i}\left\langle 0\left|\left[\hat{Y}_{\mathrm{A}}, \hat{Y}_{\mathrm{B}}\right]\right| 0\right\rangle, \\
\omega & :=2\left\langle 0\left|\left\{\hat{Y}_{\mathrm{A}}, \hat{Y}_{\mathrm{B}}\right\}\right| 0\right\rangle .
\end{aligned}
$$

These quantities $\Theta$ and $\omega$ can be considered as the PauliJordan and Hadamard distributions [40, respectively. The quantity $\Theta$ is nonzero whenever detectors can send and receive signals but vanishes otherwise (e.g., when the support of smeared detectors are spatially separated), and so it provides a measure of communication between detectors. On the other hand, $\omega$ can be nonzero even when detectors cannot communicate with each other. For this reason, it is associated with correlations between noncommunicating detectors. For example, only the Hadamard distribution contributes to entanglement harvesting with spacelike separated detectors.

\section{B. Density matrix}

Suppose the detectors are initially in the following state

$$
|\psi\rangle=\alpha\left|g_{\mathrm{A}} g_{\mathrm{B}}\right\rangle+\sqrt{1-\alpha^{2}} e^{\mathrm{i} \theta}\left|e_{\mathrm{A}} e_{\mathrm{B}}\right\rangle,
$$

where $\alpha \in[0,1]$ and $\theta \in[0,2 \pi)$ is a relative phase. Note that the state is separable if $\alpha=0$ or 1 ; otherwise it is entangled. In particular, two detectors are maximally entangled if $\alpha=1 / \sqrt{2}$.

Before proceeding, we remark that we could also consider

$$
|\tilde{\psi}\rangle=\alpha\left|g_{\mathrm{A}} e_{\mathrm{B}}\right\rangle+\sqrt{1-\alpha^{2}} e^{\mathrm{i} \theta}\left|e_{\mathrm{A}} g_{\mathrm{B}}\right\rangle
$$

to be the initial state. The effects of using $|\tilde{\psi}\rangle$ will be very similar to those we obtain from $|\psi\rangle$, since the latter state yields the former upon setting $\Omega_{B} \rightarrow-\Omega_{B}$. In what follows we shall comment on $|\tilde{\psi}\rangle$ as appropriate.

Assuming the quantum field is in the vacuum state $|0\rangle$, the reduced density matrix $\rho_{\mathrm{AB}}$ of the detectors after interacting with the field is

$$
\rho_{\mathrm{AB}}=\operatorname{Tr}_{\phi}\left[\hat{U}_{\mathrm{I}}\left(\rho_{\mathrm{AB}, 0} \otimes|0\rangle\langle 0|\right) \hat{U}_{\mathrm{I}}^{\dagger}\right]
$$




$$
=\left[\begin{array}{cccc}
r_{11} & 0 & 0 & r_{14} \\
0 & r_{22} & r_{23} & 0 \\
0 & r_{23}^{*} & r_{33} & 0 \\
r_{14}^{*} & 0 & 0 & r_{44}
\end{array}\right]
$$

where $\rho_{\mathrm{AB}, 0}=|\psi\rangle\langle\psi|$, and we choose a basis $\left\{\left|g_{\mathrm{A}} g_{\mathrm{B}}\right\rangle,\left|g_{\mathrm{A}} e_{\mathrm{B}}\right\rangle,\left|e_{\mathrm{A}} g_{\mathrm{B}}\right\rangle,\left|e_{\mathrm{A}} e_{\mathrm{B}}\right\rangle\right\}$. We make an addi- tional assumption that the detectors are inertial, without relative velocity (namely, $\left.\frac{\mathrm{d} \tau_{j}}{\mathrm{~d} t}=1\right)$, in an $(n+1)$ dimensional Minkowski spacetime, interacting with a massless and minimally coupled scalar field. Then one can derive an explicit form for the elements in the density matrix $\rho_{\mathrm{AB}}$ as (see Appendix A)

$$
\begin{aligned}
& r_{11}=\frac{1}{4}\left[1+f_{\mathrm{A}} f_{\mathrm{B}} \cosh \omega+\left(2 \alpha^{2}-1\right)\left(f_{\mathrm{A}}+f_{\mathrm{B}} \cos (2 \Theta)\right)\right]+\frac{1}{2} \alpha \sqrt{1-\alpha^{2}} f_{\mathrm{B}}\left[f_{\mathrm{A}} \sinh \omega \cos \vartheta-\sin (2 \Theta) \sin \vartheta\right] \\
& r_{22}=\frac{1}{4}\left[1-f_{\mathrm{A}} f_{\mathrm{B}} \cosh \omega+\left(2 \alpha^{2}-1\right)\left(f_{\mathrm{A}}-f_{\mathrm{B}} \cos (2 \Theta)\right)\right]-\frac{1}{2} \alpha \sqrt{1-\alpha^{2}} f_{\mathrm{B}}\left[f_{\mathrm{A}} \sinh \omega \cos \vartheta-\sin (2 \Theta) \sin \vartheta\right] \\
& r_{33}=\frac{1}{4}\left[1-f_{\mathrm{A}} f_{\mathrm{B}} \cosh \omega-\left(2 \alpha^{2}-1\right)\left(f_{\mathrm{A}}-f_{\mathrm{B}} \cos (2 \Theta)\right)\right]-\frac{1}{2} \alpha \sqrt{1-\alpha^{2}} f_{\mathrm{B}}\left[f_{\mathrm{A}} \sinh \omega \cos \vartheta+\sin (2 \Theta) \sin \vartheta\right] \\
& r_{44}=\frac{1}{4}\left[1+f_{\mathrm{A}} f_{\mathrm{B}} \cosh \omega-\left(2 \alpha^{2}-1\right)\left(f_{\mathrm{A}}+f_{\mathrm{B}} \cos (2 \Theta)\right)\right]+\frac{1}{2} \alpha \sqrt{1-\alpha^{2}} f_{\mathrm{B}}\left[f_{\mathrm{A}} \sinh \omega \cos \vartheta+\sin (2 \Theta) \sin \vartheta\right] \\
& r_{14} e^{\mathrm{i}\left(\Omega_{\mathrm{A}} \tau_{\mathrm{A}, 0}+\Omega_{\mathrm{B}} \tau_{\mathrm{B}, 0}\right)} \\
& =\frac{f_{\mathrm{B}}}{4}\left[f_{\mathrm{A}} \sinh \omega+\mathrm{i}\left(2 \alpha^{2}-1\right) \sin (2 \Theta)\right]+\frac{1}{2} \alpha \sqrt{1-\alpha^{2}}\left[\left(1+f_{\mathrm{A}} f_{\mathrm{B}} \cosh \omega\right) \cos \vartheta+\mathrm{i}\left(f_{\mathrm{A}}+f_{\mathrm{B}} \cos (2 \Theta)\right) \sin \vartheta\right] \\
& r_{23} e^{\mathrm{i}\left(\Omega_{\mathrm{A}} \tau_{\mathrm{A}, 0}+\Omega_{\mathrm{B}} \tau_{\mathrm{B}, 0}\right)} \\
& =-\frac{f_{\mathrm{B}}}{4}\left[f_{\mathrm{A}} \sinh \omega+\mathrm{i}\left(2 \alpha^{2}-1\right) \sin (2 \Theta)\right]+\frac{1}{2} \alpha \sqrt{1-\alpha^{2}}\left[\left(1-f_{\mathrm{A}} f_{\mathrm{B}} \cosh \omega\right) \cos \vartheta+\mathrm{i}\left(f_{\mathrm{A}}-f_{\mathrm{B}} \cos (2 \Theta)\right) \sin \vartheta\right] .
\end{aligned}
$$

Here

$$
\begin{aligned}
& \vartheta:=\Omega_{\mathrm{A}} \tau_{\mathrm{A}, 0}+\Omega_{\mathrm{B}} \tau_{\mathrm{B}, 0}-\theta \\
& \beta_{j}(\boldsymbol{k}):=-\mathrm{i} \frac{2 \lambda_{j} \eta_{j}}{\sqrt{2|\boldsymbol{k}|}} \tilde{F}_{j}^{*}(\boldsymbol{k}) e^{\mathrm{i}|\boldsymbol{k}| \tau_{j, 0}-\mathrm{i} \boldsymbol{k} \cdot \boldsymbol{x}_{j}}, \\
& f_{j}:=\exp \left(-\frac{1}{2} \int \mathrm{d}^{n} k\left|\beta_{j}(\boldsymbol{k})\right|^{2}\right) \in \mathbb{R} \\
& \Theta=\frac{\mathrm{i}}{4} \int \mathrm{d}^{n} k\left[\beta_{\mathrm{A}}^{*}(\boldsymbol{k}) \beta_{\mathrm{B}}(\boldsymbol{k})-\beta_{\mathrm{A}}(\boldsymbol{k}) \beta_{\mathrm{B}}^{*}(\boldsymbol{k})\right] \in \mathbb{R}, \\
& \omega=-\frac{1}{2} \int \mathrm{d}^{n} k\left[\beta_{\mathrm{A}}^{*}(\boldsymbol{k}) \beta_{\mathrm{B}}(\boldsymbol{k})+\beta_{\mathrm{A}}(\boldsymbol{k}) \beta_{\mathrm{B}}^{*}(\boldsymbol{k})\right] \in \mathbb{R},
\end{aligned}
$$

where $\tilde{F}_{j}(\boldsymbol{k})$ is the Fourier transformed smearing function.

It is worth noting that this form lets us easily examine the behavior of correlations between the detectors. For instance, there are several terms that vanish for particular cases such as $\alpha=1 / \sqrt{2}, \alpha=0,1$, or $\Theta=0$. In addition, we can consider a scenario where the detectors are initially prepared in the state $|\psi\rangle$ but only one of them interacts with the field. For example, if we want only Bob to interact then we set $\lambda_{\mathrm{A}}=0$, leading to $f_{\mathrm{A}}=1, \Theta=\omega=0$.

Finally let us specify the spatial profile of the detectors. We choose the Gaussian smearing function

$$
F(\boldsymbol{x})=\frac{1}{(\sqrt{\pi} \sigma)^{n}} e^{-\boldsymbol{x}^{2} / \sigma^{2}},
$$

with a typical Gaussian width $\sigma$. Restricting ourselves to $(3+1)$-dimensional Minkowski spacetime, we obtain (see Appendix A

$$
\begin{aligned}
& f_{j}=\exp \left(-\frac{\lambda_{j}^{2} \eta_{j}^{2}}{2 \pi^{2} \sigma^{2}}\right), \\
& \Theta=\frac{\lambda_{\mathrm{A}} \lambda_{\mathrm{B}} \eta_{\mathrm{A}} \eta_{\mathrm{B}}}{4 \pi^{2} L \sigma} \sqrt{\frac{\pi}{2}}\left(e^{-(\Delta \tau+L)^{2} / 2 \sigma^{2}}-e^{-(\Delta \tau-L)^{2} / 2 \sigma^{2}}\right), \\
& \omega=-\frac{\lambda_{\mathrm{A}} \lambda_{\mathrm{B}} \eta_{\mathrm{A}} \eta_{\mathrm{B}}}{\sqrt{2} \pi^{2} L \sigma}\left[D^{+}\left(\frac{\Delta \tau+L}{\sqrt{2} \sigma}\right)-D^{+}\left(\frac{\Delta \tau-L}{\sqrt{2} \sigma}\right)\right],
\end{aligned}
$$

where $\Delta \tau:=\tau_{\mathrm{B}, 0}-\tau_{\mathrm{A}, 0}, L:=\left|\boldsymbol{x}_{\mathrm{B}}-\boldsymbol{x}_{\mathrm{A}}\right|$, and $D^{+}(x)$ is the Dawson function defined by

$$
D^{+}(x):=\frac{\sqrt{\pi}}{2} e^{-x^{2}} \operatorname{erfi}(x)
$$

with the imaginary error function erfi $(x)$. Note that $\Delta \tau=L$ coresponds to the case when detectors are lightlike separated.

\section{Correlation measure}

We are particularly interested in how the amount of entanglement and mutual information change after the interaction. We shall quantify the entanglement between the detectors using concurrence. 
Suppose we have a density matrix $\rho_{\mathrm{AB}}$ of a joint system $\mathcal{H}_{\mathrm{A}} \otimes \mathcal{H}_{\mathrm{B}}$. The concurrence of $\rho_{\mathrm{AB}}$ is defined to be [41]

$$
\begin{array}{r}
\mathcal{C}\left(\rho_{\mathrm{AB}}\right):=\max \left\{0, w_{1}-w_{2}-w_{3}-w_{4}\right\}, \\
\left(w_{1} \geq w_{2} \geq w_{3} \geq w_{4}\right)
\end{array}
$$

where $w_{i}$ are the square roots of the eigenvalues of $\rho_{\mathrm{AB}} \tilde{\rho}_{\mathrm{AB}}$, and

$$
\tilde{\rho}_{\mathrm{AB}}:=\left(\sigma_{y} \otimes \sigma_{y}\right) \rho_{\mathrm{AB}}^{*}\left(\sigma_{y} \otimes \sigma_{y}\right) .
$$

Here $\sigma_{y}$ is the Pauli- $y$ matrix. For the density matrix (14) we get

$$
w_{i} \in\left\{\sqrt{r_{11} r_{44}} \pm\left|r_{14}\right|, \sqrt{r_{22} r_{33}} \pm\left|r_{23}\right|\right\},
$$

and so the concurrence is either

$$
\begin{array}{r}
\mathcal{C}\left(\rho_{\mathrm{AB}}\right)=2 \max \left\{0,\left|r_{14}\right|-\sqrt{r_{22} r_{33}}\right\}, \\
\left(\text { when } w_{1}=\sqrt{r_{11} r_{44}}+\left|r_{14}\right|\right) \\
\mathcal{C}\left(\rho_{\mathrm{AB}}\right)=2 \max \left\{0,\left|r_{23}\right|-\sqrt{r_{11} r_{44}}\right\} . \\
\left(\text { when } w_{1}=\sqrt{r_{22} r_{33}}+\left|r_{23}\right|\right)
\end{array}
$$

If the initial state is 11 then we always get 29). On the other hand, if we use (12) as the initial state, then the concurrence is given by (30).

Let us now define mutual information, which is a measure of total correlation including classical and quantum. The mutual information $I\left(\rho_{\mathrm{AB}}\right)$ between detectors $\mathrm{A}$ and $\mathrm{B}$ is defined to be 42

$$
I\left(\rho_{\mathrm{AB}}\right):=S\left(\rho_{\mathrm{A}}\right)+S\left(\rho_{\mathrm{B}}\right)-S\left(\rho_{\mathrm{AB}}\right),
$$

where $\rho_{\mathrm{A}}=\operatorname{Tr}_{\mathrm{B}}\left[\rho_{\mathrm{AB}}\right]$ (and vice-versa), and $S(\rho):=$ $-\operatorname{Tr}[\rho \ln \rho]$ is the von Neumann entropy.

We can evaluate mutual information explicitly from the following density matrices.

$$
\rho_{\mathrm{A}}=\left[\begin{array}{cc}
P_{\mathrm{A}}^{g} & 0 \\
0 & P_{\mathrm{A}}^{e}
\end{array}\right], \rho_{\mathrm{B}}=\left[\begin{array}{cc}
P_{\mathrm{B}}^{g} & 0 \\
0 & P_{\mathrm{B}}^{e}
\end{array}\right],
$$

where $P_{j}^{g}$ and $P_{j}^{e}$ are the probabilities of detector- $j$ being in ground and excited states, respectively, and they read

$$
\begin{aligned}
P_{\mathrm{A}}^{g}= & r_{11}+r_{22}=\frac{1}{2}\left[1+\left(2 \alpha^{2}-1\right) f_{\mathrm{A}}\right], \\
P_{\mathrm{A}}^{e}= & r_{33}+r_{44}=\frac{1}{2}\left[1-\left(2 \alpha^{2}-1\right) f_{\mathrm{A}}\right], \\
P_{\mathrm{B}}^{g}= & r_{11}+r_{33} \\
= & \frac{1}{2}\left[1+\left(2 \alpha^{2}-1\right) f_{\mathrm{B}} \cos (2 \Theta)\right] \\
& \quad-\alpha \sqrt{1-\alpha^{2}} f_{\mathrm{B}} \sin (2 \Theta) \sin \vartheta, \\
P_{\mathrm{B}}^{e}= & r_{22}+r_{44} \\
= & \frac{1}{2}\left[1-\left(2 \alpha^{2}-1\right) f_{\mathrm{B}} \cos (2 \Theta)\right] \\
& \quad+\alpha \sqrt{1-\alpha^{2}} f_{\mathrm{B}} \sin (2 \Theta) \sin \vartheta .
\end{aligned}
$$

Notice that the probabilities in $\rho_{\mathrm{A}}$ only depend on the initial state $\alpha$ and $f_{\mathrm{A}}$, while for $\rho_{\mathrm{B}}$, probabilities depend

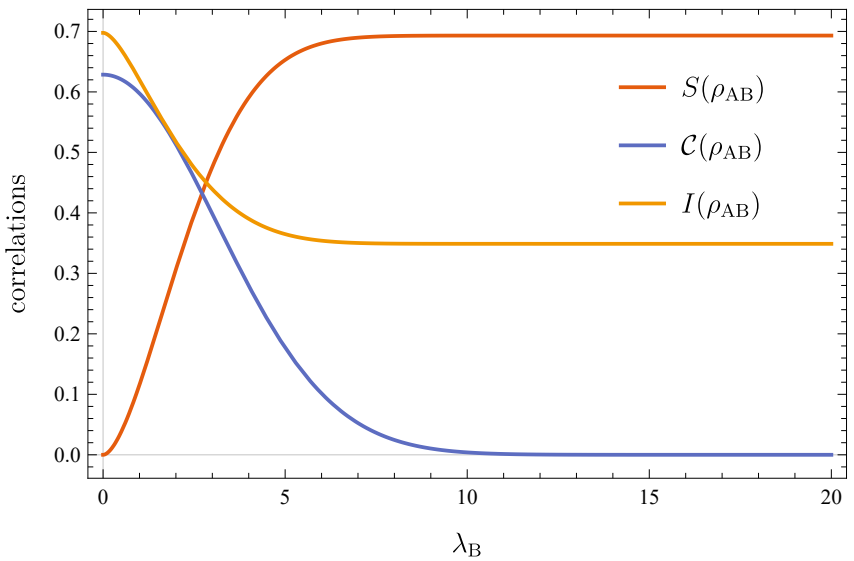

FIG. 1. The trade-off property among the correlations $S\left(\rho_{\mathrm{AB}}\right), \mathcal{C}\left(\rho_{\mathrm{AB}}\right)$, and $I\left(\rho_{\mathrm{AB}}\right)$. Here $\alpha=1 / 3, \eta / \sigma=1 . \quad \lambda_{\mathrm{B}}=0$ represents the initial correlations, and $\lambda_{\mathrm{B}} \gg 1$ corresponds to the extremely noisy scenario.

also on the commutator $\Theta$ and the phase $\vartheta$. This can be understood by recalling our assumption that Alice always turns on the detector first, i.e., $t\left(\tau_{\mathrm{A}, 0}\right) \leq t\left(\tau_{\mathrm{B}, 0}\right)$. Thus, Alice cannot be influenced by Bob but Bob could be affected by Alice by receiving signals. In fact, if there is no signal at all $(\Theta=0)$ then Bob's probabilities take the same form as Alice's.

The entropy $S\left(\rho_{\mathrm{AB}}\right)$ is computed by

$$
S\left(\rho_{\mathrm{AB}}\right)=-\sum_{i=1}^{4} p_{i} \ln p_{i}
$$

where $p_{i}$ are the eigenvalues of $\rho_{\mathrm{AB}}$,

$$
\begin{aligned}
& p_{1}=\frac{r_{11}+r_{44}}{2}+\sqrt{\left(\frac{r_{11}-r_{44}}{2}\right)^{2}+\left|r_{14}\right|^{2}}, \\
& p_{2}=\frac{r_{11}+r_{44}}{2}-\sqrt{\left(\frac{r_{11}-r_{44}}{2}\right)^{2}+\left|r_{14}\right|^{2}}, \\
& p_{3}=\frac{r_{22}+r_{33}}{2}+\sqrt{\left(\frac{r_{22}-r_{33}}{2}\right)^{2}+\left|r_{23}\right|^{2}}, \\
& p_{4}=\frac{r_{22}+r_{33}}{2}-\sqrt{\left(\frac{r_{22}-r_{33}}{2}\right)^{2}+\left|r_{23}\right|^{2}} .
\end{aligned}
$$

$S\left(\rho_{\mathrm{AB}}\right)$ is also used to measure entanglement between the field and the detectors.

\section{RESULTS}

\section{A. Single detector interacting with the field}

To begin with, let us consider the scenario where we prepare the initial state 11 and then only Bob interacts with the field. Since there no signalling effect comes 
(a)

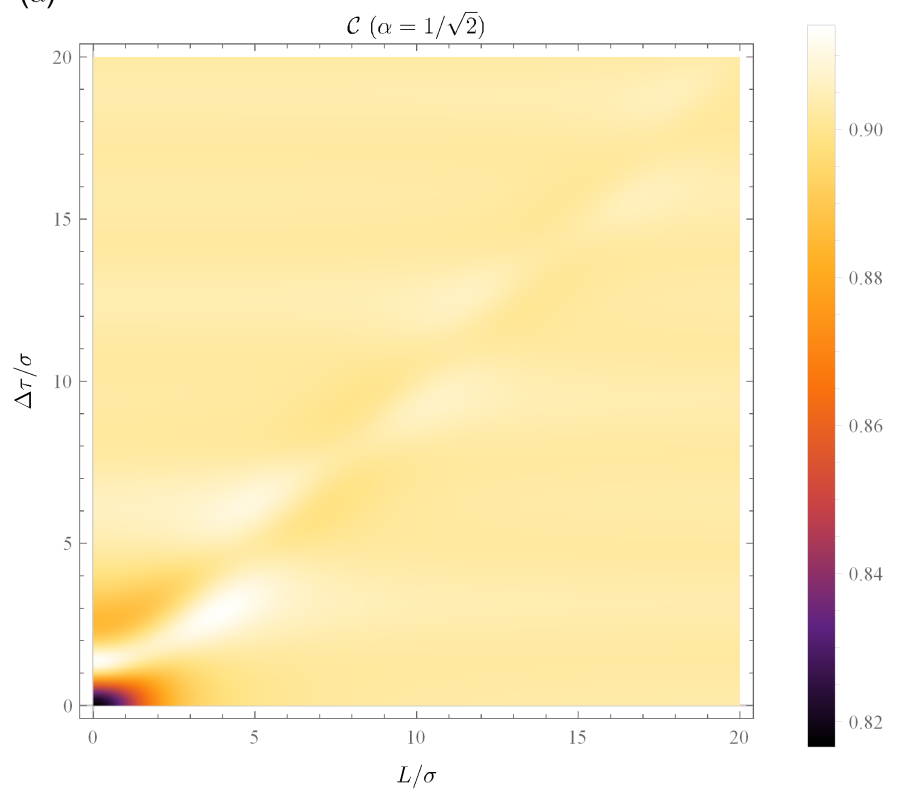

(b)

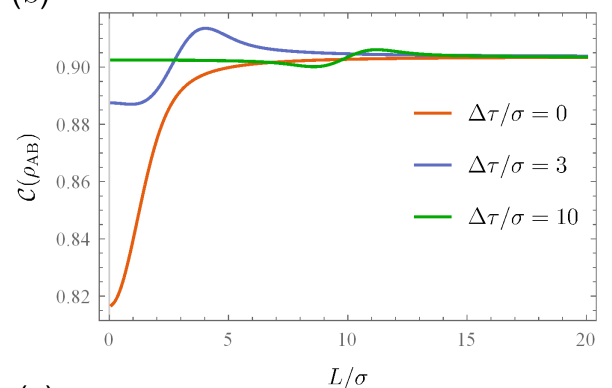

(c)

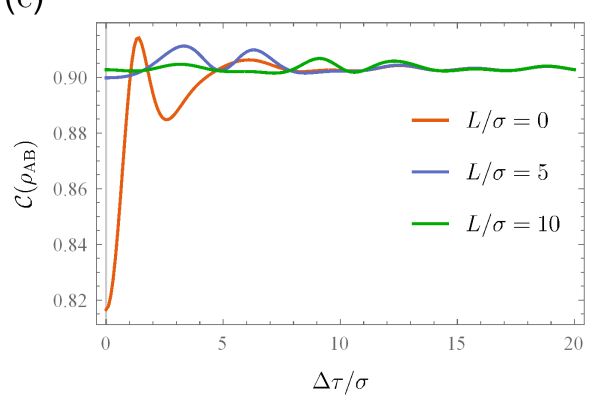

FIG. 2. (a) Concurrence as a distribution of the detector separation $L / \sigma$ and switching difference $\Delta \tau / \sigma$. Here we choose $\alpha=1 / \sqrt{2}, \theta=0, \Omega \sigma=1, \lambda=1, \eta / \sigma=1$. (b) Concurrence as a function of $L / \sigma$ at fixed $\Delta \tau / \sigma=0,3$, and 10 . The value of $\mathcal{C}$ near $L=\Delta \tau$ is disturbed due to signalling from Alice to Bob. (c) Concurrence as a function of $\Delta \tau / \sigma$ at fixed $L / \sigma=0,5$, and 10.

into play, we can examine how the initial entanglement is affected purely by the field.

We set $\lambda_{\mathrm{A}}=0$, which leads to $f_{\mathrm{A}}=1$ and $\Theta=\omega=0$; thereby the elements $15 \mathrm{a}-15 \mathrm{f}$ reduce to

$$
\begin{aligned}
& r_{11}=\frac{\alpha^{2}}{2}\left(1+f_{\mathrm{B}}\right), \\
& r_{22}=\frac{\alpha^{2}}{2}\left(1-f_{\mathrm{B}}\right), \\
& r_{33}=\frac{1-\alpha^{2}}{2}\left(1-f_{\mathrm{B}}\right), \\
& r_{44}=\frac{1-\alpha^{2}}{2}\left(1+f_{\mathrm{B}}\right), \\
& r_{14}=\frac{1}{2} \alpha \sqrt{1-\alpha^{2}}\left(1+f_{\mathrm{B}}\right) e^{-\mathrm{i} \theta}, \\
& r_{23}=\frac{1}{2} \alpha \sqrt{1-\alpha^{2}}\left(1-f_{\mathrm{B}}\right) e^{-\mathrm{i} \theta} .
\end{aligned}
$$

Note that all the elements are independent of position in a spacetime.

Concurrence $\mathcal{C}\left(\rho_{\mathrm{AB}}\right)$ and the terms in mutual information $I\left(\rho_{\mathrm{AB}}\right)$ are then

$$
\begin{aligned}
\mathcal{C}\left(\rho_{\mathrm{AB}}\right) & =2 \alpha \sqrt{1-\alpha^{2}} f_{\mathrm{B}}, \\
S\left(\rho_{\mathrm{A}}\right) & =-\alpha^{2} \ln \alpha^{2}-\left(1-\alpha^{2}\right) \ln \left(1-\alpha^{2}\right), \\
S\left(\rho_{\mathrm{B}}\right) & =-\frac{1+\left(2 \alpha^{2}-1\right) f_{\mathrm{B}}}{2} \ln \frac{1+\left(2 \alpha^{2}-1\right) f_{\mathrm{B}}}{2}
\end{aligned}
$$

$$
\begin{array}{r}
-\frac{1-\left(2 \alpha^{2}-1\right) f_{\mathrm{B}}}{2} \ln \frac{1-\left(2 \alpha^{2}-1\right) f_{\mathrm{B}}}{2}, \\
S\left(\rho_{\mathrm{AB}}\right)=-\frac{1+f_{\mathrm{B}}}{2} \ln \frac{1+f_{\mathrm{B}}}{2}-\frac{1-f_{\mathrm{B}}}{2} \ln \frac{1-f_{\mathrm{B}}}{2} .
\end{array}
$$

Observe that concurrence is proportional to $f_{\mathrm{B}} \in(0,1]$, and it is non-increasing, namely, the detectors cannot gain entanglement after Bob interacts with the field, regardless of the value of $\alpha$. In fact, the stronger the field coupling, the greater the degradation of the initial entanglement, which vanishes in the strong coupling regime as $\lambda_{\mathrm{B}} \rightarrow \infty$. Furthermore, if the detectors are initially separable $(\alpha=0,1)$ then $\mathcal{C}\left(\rho_{\mathrm{AB}}\right)=0$ for all $\lambda_{\mathrm{B}}$.

Regarding the von Neumann entropies, $S\left(\rho_{\mathrm{A}}\right)$ is constant because Alice never interacts with the field, whereas Bob's detector will obviously be affected. We see from (46) that the entanglement $S\left(\rho_{\mathrm{AB}}\right)$ between the field and the detectors only depends on $f_{\mathrm{B}}$ and not on $\alpha$. Regardless of the initial state, the entanglement between the field and the joint system of two detectors is the same.

Notice that concurrence and mutual information have a trade-off property with the field-detectors entanglement $S\left(\rho_{\mathrm{AB}}\right)$ as shown in Fig. 1. We find $\mathcal{C}\left(\rho_{\mathrm{AB}}\right)$ and $I\left(\rho_{\mathrm{AB}}\right)$ are maximum when $S\left(\rho_{\mathrm{AB}}\right)$ is minimum, and vice versa. This phenomenon can be thought of as the leakage of initial detector correlations to the quantum field.

Although the concurrence vanishes at large $\lambda_{\mathrm{B}}$, Fig. 1 indicates that mutual information is always nonzero. 

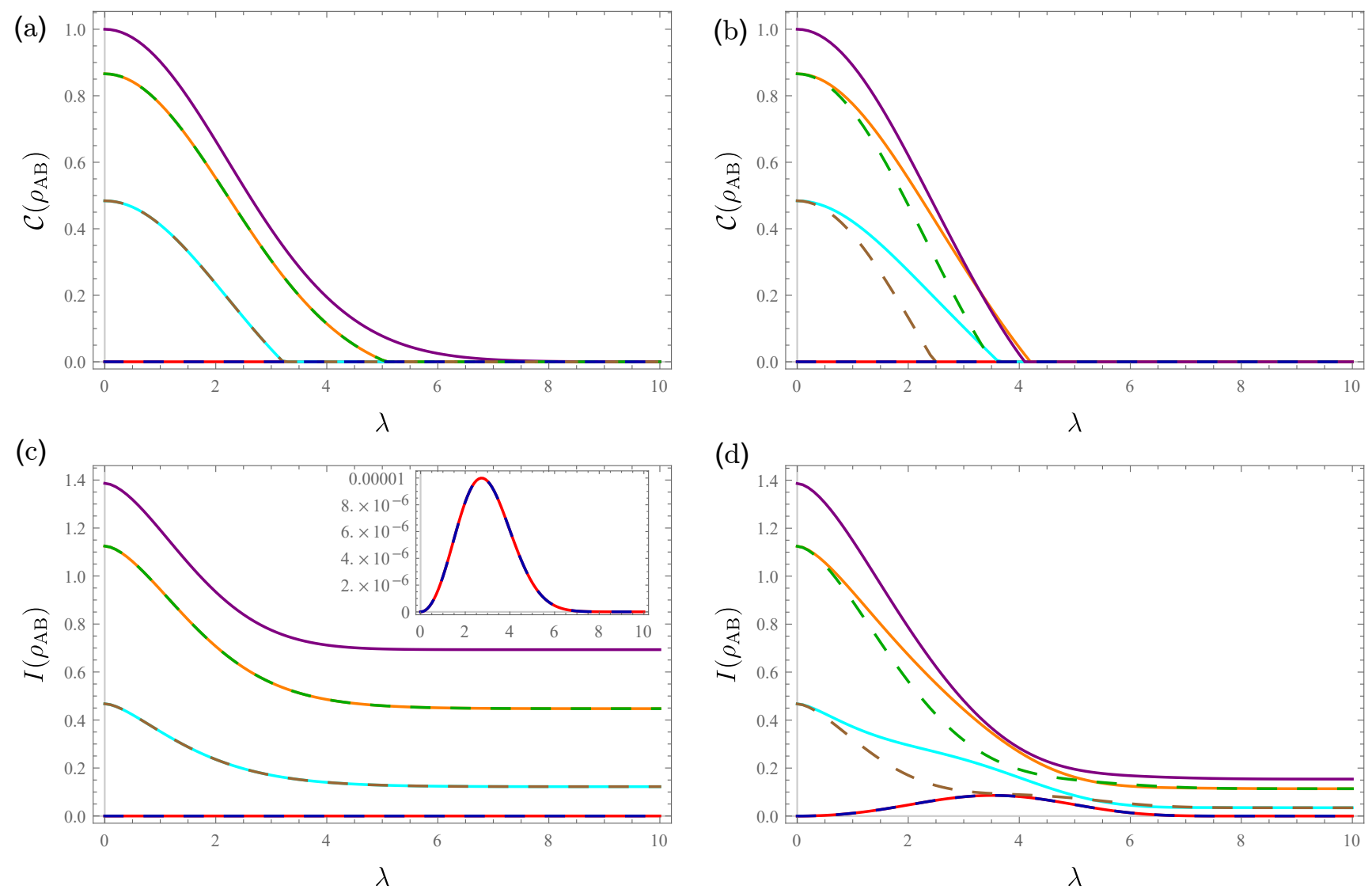

$$
\begin{aligned}
& -\alpha=0,-\alpha=1 / 4,-\alpha=1 / 2,-\alpha=1 / \sqrt{2}, \\
& -\alpha=\sqrt{3} / 2,-\alpha=\sqrt{15} / 4,-\alpha=1
\end{aligned}
$$

FIG. 3. Concurrence (a), (b), and mutual information (c), (d) as a function of coupling strength $\lambda$ when the detectors are (a), (c) spacelike $L / \sigma=10, \tau_{\mathrm{A}, 0}=\tau_{\mathrm{B}, 0}=0$ and (b), (d) lightlike $L / \sigma=\Delta \tau / \sigma=1$, with $\theta=0, \Omega \sigma=1, \eta / \sigma=1$ for all cases. Even though entanglement cannot be harvested, mutual information can be. Also, lightlike separated detectors have different degradation properties with different $\alpha$ although the initial amount of entanglement is the same.

By setting $\lambda_{\mathrm{B}} \rightarrow \infty, f_{\mathrm{B}}$ becomes 0 , and we get $S\left(\rho_{\mathrm{B}}\right)=$ $S\left(\rho_{\mathrm{AB}}\right)=\ln 2$. Hence the mutual information in the strong coupling regime becomes $I\left(\rho_{\mathrm{AB}}\right)=S\left(\rho_{\mathrm{A}}\right)$, which is nonzero unless $\alpha=0,1$. This is exactly half the value of the initial mutual information, which can be evaluated by substituting $\lambda_{\mathrm{B}}=0$, i.e., $f_{\mathrm{B}}=1$, yielding $I\left(\rho_{\mathrm{AB}}\right)=2 S\left(\rho_{\mathrm{A}}\right)$. This is similar to situations in which initially entangled modes become unentangled from the viewpoint of a uniformly accelerating observer in Minkowski spacetime in the large acceleration limit 27, or to a static observer in a spherically symmetric black hole spacetime with infinite Hawking temperature, while mutual information is degraded to half of its initial value [28, 43,44 .

\section{B. Both detectors interacting with the field}

We now consider two detectors interacting with the field. In what follows, all the values are in units of the typical Gaussian smearing width $\sigma$.

\section{Entanglement degradation}

To begin with, let us look at the concurrence in a spacetime diagram. Figure 2 shows the concurrence when maximally entangled detectors are initially prepared. Figure 2(a) is a density plot of $\mathcal{C}\left(\rho_{\mathrm{AB}}\right)$ in terms of the detector separation $L / \sigma$ and the switching difference $\Delta \tau / \sigma$, with $\theta=0, \Omega \sigma=1, \lambda=1, \eta / \sigma=1$. Given that the concurrence of the maximally entangled state is $\mathcal{C}\left(\rho_{\mathrm{AB}}\right)=1$, one immediately notices that the entanglement is degraded under all circumstances. Entanglement experiences its greatest degradation for small values of 
(a)

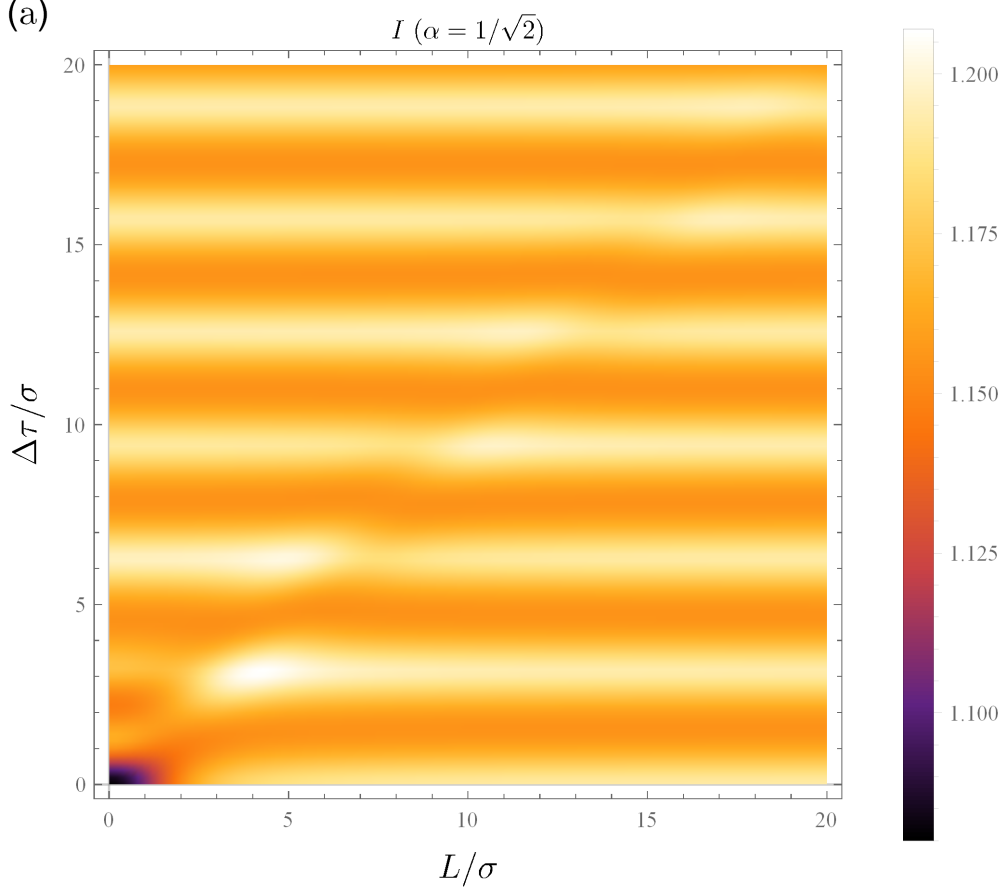

(b)

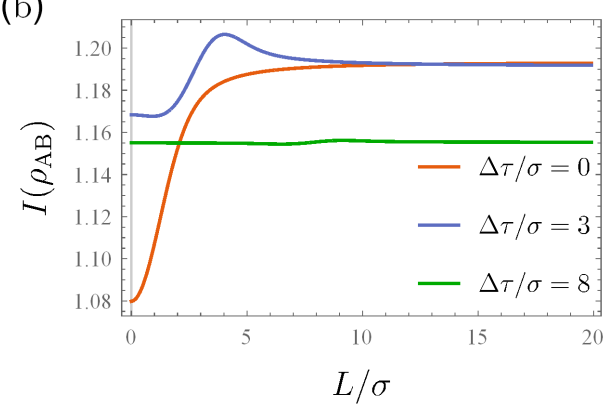

(c)

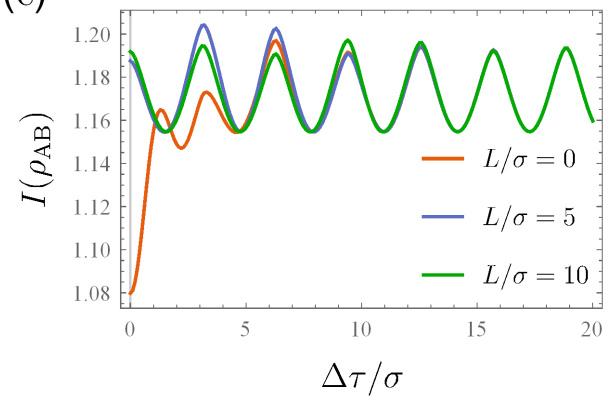

FIG. 4. (a) Mutual information as a distribution of the detector separation $L / \sigma$ and switching difference $\Delta \tau / \sigma$. Here we choose $\alpha=1 / \sqrt{2}, \theta=0, \Omega \sigma=1, \lambda=1$, and $\eta / \sigma=1$. (b) Mutual information as a function of $L / \sigma$ at fixed $\Delta \tau / \sigma=0,3$, and 8. (c) Mutual information as a function of $\Delta \tau / \sigma$ at fixed $L / \sigma=0,5$, and 10.

$L / \sigma$ and $\Delta \tau / \sigma$, but asymptotes to about $90 \%$ of its original value for large values of these quantities. We also notice that there is a pattern in the figure; the amount of entanglement changes periodically with $\Delta \tau / \sigma$ due to the phase factor $\vartheta$. This is more evident in Fig. 2 (c), which is a plot of concurrence as a function of $\Delta \tau / \sigma$ with fixed detector separation $L / \sigma$.

Another characteristic can be seen when detectors are nearly lightlike separated, with $L=\Delta \tau$. In this case the concurrence shows a distorting oscillation due to the exchange of quanta. This is clear from Fig. 2(b), where concurrence is plotted as a function of separation $L / \sigma$ with fixed $\Delta \tau / \sigma$. The concurrence is distorted in a region near $L=\Delta \tau$, but becomes constant for large separation $L / \sigma$. This suggests that communication affects the amount of entanglement degradation.

Let us now examine how field interactions affect the initial entanglement. Figures 3 (a) and (b) depict concurrence as a function of the coupling strength $\lambda$ when detectors are (a) spacelike separated $L / \sigma=10, \tau_{\mathrm{A}, 0} / \sigma=$ $\tau_{\mathrm{B}, 0} / \sigma=0$ and (b) lightlike separated $L / \sigma=\Delta \tau / \sigma=1$. In both cases, we set $\theta=0, \Omega \sigma=1$, and $\eta / \sigma=1$.

Both scenarios show that entanglement is nonincreasing, vanishing at some value of $\lambda$. Since $\lambda=0$ implies that there is no interaction between the field and the detectors, the value of $\mathcal{C}\left(\rho_{\mathrm{AB}}\right)$ at $\lambda=0$ is the initial amount of entanglement. Note that initially separable detectors $(\alpha=0,1)$ cannot harvest entanglement from the vacuum even if they are communicationassisted. This is consistent with earlier studies [11, 12] that found delta-switched detectors cannot harvest entanglement from the field in a coherent state as well as a vacuum state.

We observe that communication modifies degradation; even if the detectors have the same amount of initial entanglement, degradation changes under swapping $\alpha$ and $\sqrt{1-\alpha^{2}}$ in the lightlike separated case. Recall that our initial entangled state was given in (11). The initial concurrence $\mathcal{C}\left(\rho_{\mathrm{AB}}\right)=2 \alpha \sqrt{1-\alpha^{2}}$ does not change under $\alpha \rightarrow \sqrt{1-\alpha^{2}}$. This is also true for spacelike separated detectors [Fig. 3(a)] but not for lightlike separated ones [Fig. 3(b)].

This effect can be verified by looking at the elements of the density matrix (15a)-(15f). Since $2 \alpha^{2}-1$ changes its sign under $\alpha \rightarrow \sqrt{1-\alpha^{2}}$, all elements will be modified. However, if the detectors cannot communicate, i.e., $\Theta=0$, then $r_{14}$ and a product $r_{22} r_{33}$ are invariant under such a transformation. Therefore, concurrence $\mathcal{C}\left(\rho_{\mathrm{AB}}\right)$ changes under $\alpha \rightarrow \sqrt{1-\alpha^{2}}$ when $\Theta \neq 0$. This kind of phenomenon has also been seen when one detector is 
noninertial [28, 43, 44].

\section{Mutual information}

We will now look at mutual information $I\left(\rho_{\mathrm{AB}}\right)$. We first show in Fig. 4 how $I\left(\rho_{\mathrm{AB}}\right)$ behaves in a spacetime when the detectors are initially maximally entangled. The behavior is similar to that of concurrence; it has periodic phase dependence and there is distortion of $I\left(\rho_{\mathrm{AB}}\right)$ when the detectors can communicate. In addition, the coupling dependence shown in Figs. 3(c) and (d) are also similar to the concurrence case in Figs. 3(a) and (b); signalling modifies the degradation property un$\operatorname{der} \alpha \rightarrow \sqrt{1-\alpha^{2}}$.

There are two notable departures from the behavior of the concurrence. One is the ability to harvest mutual information. The other is that of nonvanishing correlation in the strong coupling limit $\lambda \rightarrow \infty$.

As shown in Figs. 3(c) and (d) with $\alpha=0$ and 1, detectors can harvest mutual information from the vacuum, even when the detectors cannot communicate with each other. Figure 5 depicts the mutual information in terms of detector separation when they are initially in their ground states $\left|g_{\mathrm{A}} g_{\mathrm{B}}\right\rangle$. One can numerically confirm that setting $\alpha=0,1$ and $\Theta=0$ still gives nonzero mutual information, indicating that noncommunicating detectors can harvest mutual information. Since entanglement is always zero, the content of this harvested correlation is classical correlation or nondistillable entanglement.

From the degradation point of view, the fact that detectors can harvest mutual information suggests that we can gain some correlation by interacting with a field. In fact, slightly entangled detectors can gain mutual information from the vacuum, which is shown in Fig. 6. This figure shows that spacelike separated, slightly entangled detectors can acquire mutual information from the vacuum state; over some range of $\lambda$, the amount of mutual information is greater than the initial amount, which is given at $\lambda=0$. This suggests that there is a case where the anti-commutator $\omega$ enhances correlations between the detectors. Note that this kind of mutual information acquisition never happens if only one detector interacts with the field, as shown in Fig. 1. because $\omega=0$. In addition, more strongly entangled detectors cannot gain correlation as shown in Fig. 3(c).

The second characteristic we observe is that mutual information is nonvanishing at large $\lambda$ [Figs. 3(c), (d)] in contrast to entanglement [Figs. 3(a), (b)]. This phenomenon has been previously observed in other contexts [27, 29, 32, 43, 44. Since entanglement is zero as $\lambda \rightarrow \infty$ but nonzero for mutual information, the remaining correlations in the strong coupling regime are classical correlations and nondistillable entanglement.

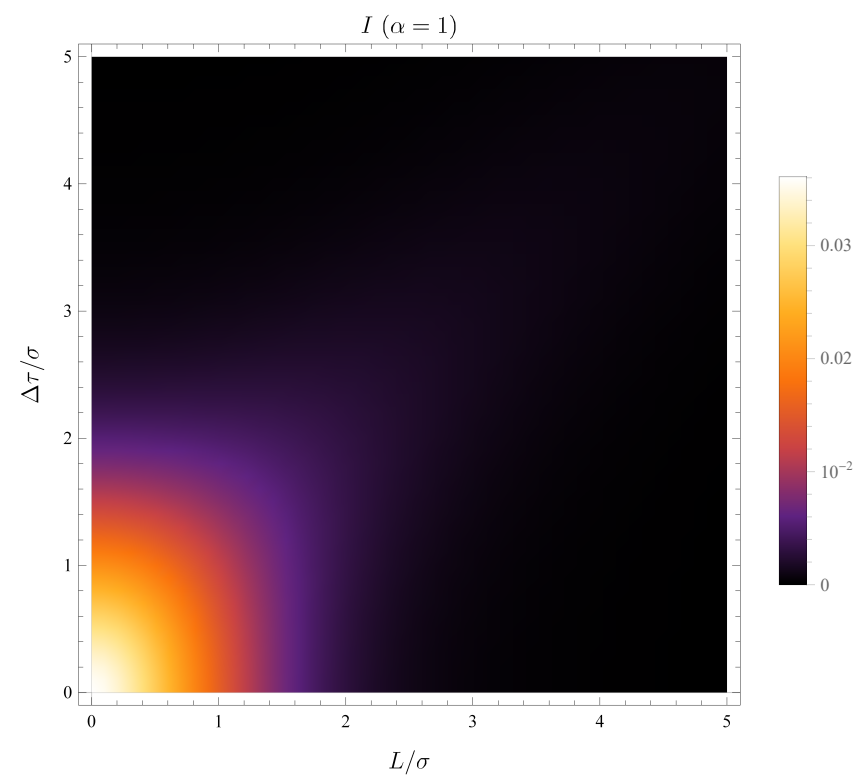

FIG. 5. A density plot of mutual information when the detectors are initially a separable state. Here we choose $\lambda=1, \Omega \sigma=1, \eta / \sigma=1$, and $\theta=0$. Unlike entanglement, detectors can harvest mutual information from the vacuum using delta switching.

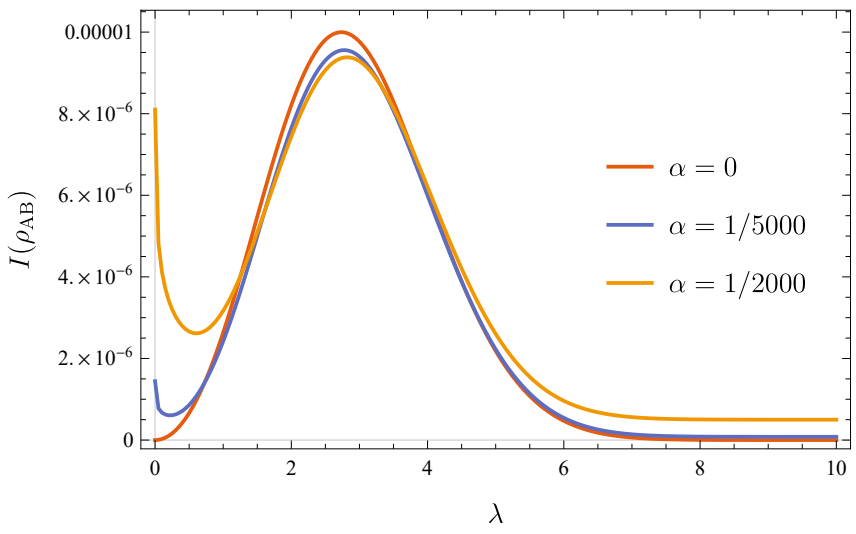

FIG. 6. Mutual information acquisition can happen for initially separable, or weakly entangled detectors. The red, blue and orange lines represent $\alpha=0,1 / 5000$, and $1 / 2000$, respectively. Here we set $\tau_{\mathrm{A}, 0} / \sigma=\tau_{\mathrm{B}, 0} / \sigma=0, L / \sigma=10, \Omega \sigma=$ $1, \eta / \sigma=1$, and $\theta=0$. For some range of $\lambda$, the detectors can gain mutual information from the vacuum compared to the initial state $(\lambda=0)$.

\section{CONCLUSION}

We have considered entanglement degradation of two initially entangled, inertial UDW detectors coupled to a scalar field in a $(3+1)$-dimensional Minkowski spacetime, employing nonperturbative methods [11. In contrast to situations in which one of the UDW detectors is accelerating, we only have considered inertial detectors. We are particularly interested in the effects the quantum vacuum 
has on detector entanglement (analogous to the entanglement harvesting protocol), and how communication channels via the field affect this.

We investigated the amount of entanglement for two cases: a single detector interacting with the field after the entangled detectors are prepared, and both detectors interacts with the field. In each case the concurrence monotonically decreased and vanished for large coupling strength $\lambda$. In this sense, detectors with delta switching experience entanglement degradation. Note that while signalling indeed affects the degradation property, it does not assist in acquiring entanglement.

Our results are complementary to those of Ref. [12, in which a general no-go theorem was demonstrated that gave constraints on whether or not a separable bipartite system of finite dimension (e.g., detectors) could become entangled through local interactions with a source that was either finite or infinite dimensional (e.g., a quantum field). The constraints of this theorem do not apply in the present case, since our bipartite system of finite dimension (the two detectors) is initially entangled.

The overall behavior of mutual information is similar to concurrence, with the important exception that weakly entangled detectors can gain mutual information for some range of values of $\lambda$. As long as the detectors are initially entangled, the mutual information never vanishes at the strong coupling regime.

Although the possibility of communication does not assist entanglement acquisition, it modifies the degradation properties of both entanglement and mutual information. We showed that two states $\alpha\left|g_{\mathrm{A}} g_{\mathrm{B}}\right\rangle+e^{\mathrm{i} \theta} \sqrt{1-\alpha^{2}}\left|e_{\mathrm{A}} e_{\mathrm{B}}\right\rangle$ and $\sqrt{1-\alpha^{2}}\left|g_{\mathrm{A}} g_{\mathrm{B}}\right\rangle+e^{\mathrm{i} \theta} \alpha\left|e_{\mathrm{A}} e_{\mathrm{B}}\right\rangle$, which are related by the transformation $\alpha \rightarrow \sqrt{1-\alpha^{2}}$, experience different rates of degradation if and only if the two detectors can communicate, even though they possess the same amount of initial entanglement.

We close by commenting on what would happen if we employed the initial state (12). Such a state can be created from (11) by passing it through the bit-flip gate $\hat{I}_{\mathrm{A}} \otimes \hat{\sigma}_{x}$, where $\hat{\sigma}_{x}$ is the Pauli- $x$ matrix acting on detector $\mathrm{B}$. The resulting final density matrix of the detectors is

$$
\left[\begin{array}{cccc}
r_{22}^{\prime} & 0 & 0 & r_{23}^{\prime} \\
0 & r_{11}^{\prime} & r_{14}^{\prime} & 0 \\
0 & \left(r_{14}^{\prime}\right)^{*} & r_{44}^{\prime} & 0 \\
\left(r_{23}^{\prime}\right)^{*} & 0 & 0 & r_{33}^{\prime}
\end{array}\right]
$$

where $r_{i j}^{\prime}$ are equivalent to $r_{i j}$ in 14 with $\Omega_{\mathrm{B}} \rightarrow-\Omega_{\mathrm{B}}$. Therefore, the overall behavior of the entanglement and mutual information is essentially the same as $\alpha\left|g_{\mathrm{A}} g_{\mathrm{B}}\right\rangle+$ $e^{\mathrm{i} \theta} \sqrt{1-\alpha^{2}}\left|e_{\mathrm{A}} e_{\mathrm{B}}\right\rangle$ case.

\section{ACKNOWLEDGMENT}

The authors thank Ahmed Shalabi and Laura J. Henderson for useful information regarding the nonperturbative calculations, and Erickson Tjoa, Eduardo MartínMartínez and Petar Simidzija for discussion and clarification on the no-go theorem. K.G-Y. acknowledges the support from Keio University Global Fellowship. This work was supported in part by the Natural Sciences and Engineering Research Council of Canada and by Asian Office of Aerospace Research and Development Grant No. FA2386-19-1-4077.

\section{Appendix A: THE ELEMENTS IN THE DENSITY MATRIX}

Let us consider two detectors in an $(n+1)$-dimensional Minkowski spacetime. The time-evolution operator $\hat{U}_{\mathrm{I}}$ given in (7) can be written as [11]

$$
\begin{aligned}
\hat{U}_{\mathrm{I}} & =\hat{I}_{\mathrm{A}} \otimes \hat{I}_{\mathrm{B}} \otimes \hat{X}_{(+,+)}+\hat{\mu}_{\mathrm{A}} \otimes \hat{I}_{\mathrm{B}} \otimes \hat{X}_{(+,-)} \\
& +\hat{I}_{\mathrm{A}} \otimes \hat{\mu}_{\mathrm{B}} \otimes \hat{X}_{(-,+)}+\hat{\mu}_{\mathrm{A}} \otimes \hat{\mu}_{\mathrm{B}} \otimes \hat{X}_{(-,-)},
\end{aligned}
$$

where

$$
\hat{X}_{(j, k)}:=\frac{1}{4}\left(e^{\hat{Y}_{\mathrm{B}}}+j e^{-\hat{Y}_{\mathrm{B}}}\right)\left(e^{\hat{Y}_{\mathrm{A}}}+k e^{-\hat{Y}_{\mathrm{A}}}\right),
$$

and we denote $\hat{X}_{( \pm, \pm)}=\hat{X}_{( \pm 1, \pm 1)}$.

Assuming that the initial state is $(11)$, the final density matrix of the detectors takes the form

$$
\begin{aligned}
\rho_{\mathrm{AB}} & =\alpha^{2} \rho_{\mathrm{AB}}^{g g}+\alpha \sqrt{1-\alpha^{2}} e^{-\mathrm{i} \theta} \rho_{\mathrm{AB}}^{g e} \\
& +\alpha \sqrt{1-\alpha^{2}} e^{\mathrm{i} \theta} \rho_{\mathrm{AB}}^{e g}+\left(1-\alpha^{2}\right) \rho_{\mathrm{AB}}^{e e},
\end{aligned}
$$

where

$$
\begin{gathered}
\rho_{\mathrm{AB}}^{\alpha \beta}:=\operatorname{Tr}_{\phi}\left[\hat{U}_{\mathrm{I}}\left(\left|\alpha_{\mathrm{A}}\right\rangle\left\langle\beta_{\mathrm{A}}|\otimes| \alpha_{\mathrm{B}}\right\rangle\left\langle\beta_{\mathrm{B}}|\otimes| 0\right\rangle\langle 0|\right) \hat{U}_{\mathrm{I}}^{\dagger}\right], \\
\alpha, \beta \in\{g, e\} .
\end{gathered}
$$

For instance, $\rho_{\mathrm{AB}}^{g g}$ reads

$$
\begin{aligned}
\rho_{\mathrm{AB}}^{g g} & =\operatorname{Tr}_{\phi}\left[\hat{U}_{\mathrm{I}}\left(\left|g_{\mathrm{A}}\right\rangle\left\langle g_{\mathrm{A}}|\otimes| g_{\mathrm{B}}\right\rangle\left\langle g_{\mathrm{B}}|\otimes| 0\right\rangle\langle 0|\right) \hat{U}_{\mathrm{I}}^{\dagger}\right] \\
& =f_{(++++)}|g g\rangle\left\langle g g\left|+f_{(+-++)} e^{-\mathrm{i} \Omega_{\mathrm{A}} \tau_{\mathrm{A}, 0}}\right| g g\right\rangle\langle e g| \\
& +f_{(-+++)} e^{-\mathrm{i} \Omega_{\mathrm{B}} \tau_{\mathrm{B}, 0}}|g g\rangle\left\langle g e\left|+f_{(--++)} e^{-\mathrm{i} \Omega_{\mathrm{A}} \tau_{\mathrm{A}, 0}} e^{-\mathrm{i} \Omega_{\mathrm{B}} \tau_{\mathrm{B}, 0}}\right| g g\right\rangle\langle e e| \\
& +f_{(+++-)} e^{\mathrm{i} \Omega_{\mathrm{A}} \tau_{\mathrm{A}, 0}}|e g\rangle\left\langle g g\left|+f_{(+-++)}\right| e g\right\rangle\langle e g|
\end{aligned}
$$




$$
\begin{aligned}
& +f_{(-++-)} e^{\mathrm{i} \Omega_{\mathrm{A}} \tau_{\mathrm{A}, 0}} e^{-\mathrm{i} \Omega_{\mathrm{B}} \tau_{\mathrm{B}, 0}}|e g\rangle\left\langle g e\left|+f_{(--+-)} e^{-\mathrm{i} \Omega_{\mathrm{B}} \tau_{\mathrm{B}, 0}}\right| e g\right\rangle\langle e e| \\
& +f_{(++-+)} e^{\mathrm{i} \Omega_{\mathrm{B}} \tau_{\mathrm{B}, 0}}|g e\rangle\left\langle g g\left|+f_{(+--+)} e^{-\mathrm{i} \Omega_{\mathrm{A}} \tau_{\mathrm{A}, 0}} e^{\mathrm{i} \Omega_{\mathrm{B}} \tau_{\mathrm{B}, 0}}\right| g e\right\rangle\langle e g| \\
& +f_{(-+-+)}|g e\rangle\left\langle g e\left|+f_{(---+)} e^{-\mathrm{i} \Omega_{\mathrm{A}} \tau_{\mathrm{A}, 0}}\right| g e\right\rangle\langle e e| \\
& +f_{(++--)} e^{\mathrm{i} \Omega_{\mathrm{A}} \tau_{\mathrm{A}, 0}} e^{\mathrm{i} \Omega_{\mathrm{B}} \tau_{\mathrm{B}, 0}}|e e\rangle\left\langle g g\left|+f_{(+---)} e^{\mathrm{i} \Omega_{\mathrm{B}} \tau_{\mathrm{B}, 0}}\right| e e\right\rangle\langle e g| \\
& +f_{(-+--)} e^{\mathrm{i} \Omega_{\mathrm{A}} \tau_{\mathrm{A}, 0}}|e e\rangle\left\langle g e\left|+f_{(----)}\right| e e\right\rangle\langle e e|
\end{aligned}
$$

where

$$
\begin{aligned}
f_{(j k l m)} & :=\left\langle 0\left|\hat{X}_{(j, k)}^{\dagger} \hat{X}_{(l, m)}\right| 0\right\rangle \\
& =(1+j l+k m+j k l m)+k(1+j l)\left\langle 0\left|e^{2 \hat{Y}_{\mathrm{A}}}\right| 0\right\rangle+m(1+j l)\left\langle 0\left|e^{-2 \hat{Y}_{\mathrm{A}}}\right| 0\right\rangle \\
& +l\langle 0| e^{-\hat{Y}_{\mathrm{A}}} e^{-2 \hat{Y}_{\mathrm{B}}} e^{\hat{Y}_{\mathrm{A}}}+j\left\langle 0\left|e^{-\hat{Y}_{\mathrm{A}}} e^{2 \hat{Y}_{\mathrm{B}}} e^{\hat{Y}_{\mathrm{A}}}\right| 0\right\rangle+k l\left\langle 0\left|e^{\hat{Y}_{\mathrm{A}}} e^{-2 \hat{Y}_{\mathrm{B}}} e^{\hat{Y}_{\mathrm{A}}}\right| 0\right\rangle \\
& +j k\left\langle 0\left|e^{\hat{Y}_{\mathrm{A}}} e^{2 \hat{Y}_{\mathrm{B}}} e^{\hat{Y}_{\mathrm{A}}}\right| 0\right\rangle+\ln \left\langle 0\left|e^{-\hat{Y}_{\mathrm{A}}} e^{-2 \hat{Y}_{\mathrm{B}}} e^{-\hat{Y}_{\mathrm{A}}}\right| 0\right\rangle+j m\left\langle 0\left|e^{-\hat{Y}_{\mathrm{A}}} e^{2 \hat{Y}_{\mathrm{B}}} e^{-\hat{Y}_{\mathrm{A}}}\right| 0\right\rangle \\
& +k l m\left\langle 0\left|e^{\hat{Y}_{\mathrm{A}}} e^{-2 \hat{Y}_{\mathrm{B}}} e^{-\hat{Y}_{\mathrm{A}}}\right| 0\right\rangle+k j m\left\langle 0\left|e^{\hat{Y}_{\mathrm{A}}} e^{2 \hat{Y}_{\mathrm{B}}} e^{-\hat{Y}_{\mathrm{A}}}\right| 0\right\rangle .
\end{aligned}
$$

$f_{(j k l m)}$ can be simplified by using the Baker-Campbell-Hausdorff formula

$$
e^{\hat{A}} e^{\hat{B}}=\exp \left(\hat{A}+\hat{B}+\frac{1}{2}[\hat{A}, \hat{B}]+\frac{1}{12}[\hat{A},[\hat{A}, \hat{B}]]-\frac{1}{12}[\hat{B},[\hat{A}, \hat{B}]]+\cdots\right) .
$$

Given that $\left[\hat{Y}_{\mathrm{A}}, \hat{Y}_{\mathrm{B}}\right]=\mathrm{i} \Theta$, we get

$$
e^{p \hat{Y}_{\mathrm{A}}} e^{q \hat{Y}_{\mathrm{B}}} e^{r \hat{Y}_{\mathrm{A}}}=e^{\mathrm{i} \Theta q(p-r) / 2} e^{(p+r) \hat{Y}_{\mathrm{A}}+q \hat{Y}_{\mathrm{B}}}, \quad(p, q, r \in \mathbb{R})
$$

Hence, the vacuum expectation values in $f_{(j k l m)}$ will be reduced to one of the following [11.

$$
\begin{aligned}
& f_{j}:=\left\langle 0\left|e^{2 \hat{Y}_{j}}\right| 0\right\rangle=\exp \left(-\frac{1}{2} \int \mathrm{d}^{n} k\left|\beta_{j}(\boldsymbol{k})\right|^{2}\right), \\
& f_{\mathrm{p}}:=\left\langle 0\left|e^{2 \hat{Y}_{\mathrm{B}}} e^{2 \hat{Y}_{\mathrm{A}}}\right| 0\right\rangle=\exp \left[-\frac{1}{2} \int \mathrm{d}^{n} k\left(\left|\beta_{\mathrm{A}}(\boldsymbol{k})\right|^{2}+\left|\beta_{\mathrm{B}}(\boldsymbol{k})\right|^{2}+2 \beta_{\mathrm{A}}(\boldsymbol{k}) \beta_{\mathrm{B}}^{*}(\boldsymbol{k})\right)\right]=f_{\mathrm{A}} f_{\mathrm{B}} e^{\omega-2 \mathrm{i} \Theta}, \\
& f_{\mathrm{m}}:=\left\langle 0\left|e^{2 \hat{Y}_{\mathrm{B}}} e^{-2 \hat{Y}_{\mathrm{A}}}\right| 0\right\rangle=\exp \left[-\frac{1}{2} \int \mathrm{d}^{n} k\left(\left|\beta_{\mathrm{A}}(\boldsymbol{k})\right|^{2}+\left|\beta_{\mathrm{B}}(\boldsymbol{k})\right|^{2}-2 \beta_{\mathrm{A}}(\boldsymbol{k}) \beta_{\mathrm{B}}^{*}(\boldsymbol{k})\right)\right]=f_{\mathrm{A}} f_{\mathrm{B}} e^{-\omega+2 \mathrm{i} \Theta},
\end{aligned}
$$

where $\omega:=2\left\langle 0\left|\left\{\hat{Y}_{\mathrm{A}}, \hat{Y}_{\mathrm{B}}\right\}\right| 0\right\rangle$ is the vacuum expectation value of the anti-commutator. Therefore, $f_{(j k l m)}$ becomes

$$
\begin{aligned}
f_{(j k l m)}=\frac{1}{16}( & (1+j l+k m+j k l m)+(1+j l)(k+m) f_{\mathrm{A}} \\
& \left.+\left[(l+j k m) e^{2 \mathrm{i} \Theta}+(j+k l m) e^{-2 \mathrm{i} \Theta}\right] f_{\mathrm{B}}+\left[(j k+l m) e^{\omega}+(j m+k l) e^{-\omega}\right] f_{\mathrm{A}} f_{\mathrm{B}}\right) .
\end{aligned}
$$

From this, notice that $f_{(+++-)}=f_{(++-+)}=f_{(+-++)}=f_{(-+++)}=f_{(---+)}=f_{(--+-)}=f_{(-+--)}=f_{(+---)}=0$. This fact simplifies $\rho_{\mathrm{AB}}^{g g}$, and by using the basis $\left\{\left|g_{\mathrm{A}} g_{\mathrm{B}}\right\rangle,\left|g_{\mathrm{A}} e_{\mathrm{B}}\right\rangle,\left|e_{\mathrm{A}} g_{\mathrm{B}}\right\rangle,\left|e_{\mathrm{A}} e_{\mathrm{B}}\right\rangle\right\}$, we get

$$
\rho_{\mathrm{AB}}^{g g}=\left[\begin{array}{cccc}
r_{11}^{g g} & 0 & 0 & r_{14}^{g g} \\
0 & r_{22}^{g g} & r_{23}^{g g} & 0 \\
0 & \left(r_{23}^{g g}\right)^{*} & r_{33}^{g g} & 0 \\
\left(r_{14}^{g g}\right)^{*} & 0 & 0 & r_{44}^{g g}
\end{array}\right],
$$

where

$$
\begin{aligned}
r_{11}^{g g} & =f_{(++++)}, \\
r_{14}^{g g} & =f_{(--++)} e^{-\mathrm{i}\left(\Omega_{\mathrm{A}} \tau_{\mathrm{A}, 0}+\Omega_{\mathrm{B}} \tau_{\mathrm{B}, 0}\right)}, \\
r_{22}^{g g} & =f_{(-+-+)}, \\
r_{23}^{g g} & =f_{(+--+)} e^{-\mathrm{i}\left(\Omega_{\mathrm{A}} \tau_{\mathrm{A}, 0}-\Omega_{\mathrm{B}} \tau_{\mathrm{B}, 0}\right)},
\end{aligned}
$$




$$
\begin{aligned}
& r_{33}^{g g}=f_{(+-+-)}, \\
& r_{44}^{g g}=f_{(----)} .
\end{aligned}
$$

With $\rho_{\mathrm{AB}}^{g e}, \rho_{\mathrm{AB}}^{e g}, \rho_{\mathrm{AB}}^{e e}$, the total density matrix becomes

$$
\rho_{\mathrm{AB}}=\left[\begin{array}{cccc}
r_{11} & 0 & 0 & r_{14} \\
0 & r_{22} & r_{23} & 0 \\
0 & r_{23}^{*} & r_{33} & 0 \\
r_{14}^{*} & 0 & 0 & r_{44}
\end{array}\right]
$$

where

$$
\begin{aligned}
& r_{11}=\alpha^{2} f_{(++++)}+\alpha \sqrt{1-\alpha^{2}} e^{-\mathrm{i} \theta} f_{(--++)} e^{\mathrm{i}\left(\Omega_{\mathrm{A}} \tau_{\mathrm{A}, 0}+\Omega_{\mathrm{B}} \tau_{\mathrm{B}, 0}\right)} \\
& +\alpha \sqrt{1-\alpha^{2}} e^{\mathrm{i} \theta} f_{(++--)} e^{-\mathrm{i}\left(\Omega_{\mathrm{A}} \tau_{\mathrm{A}, 0}+\Omega_{\mathrm{B}} \tau_{\mathrm{B}, 0}\right)}+\left(1-\alpha^{2}\right) f_{(----)}, \\
& r_{14}=\alpha^{2} f_{(--++)} e^{-\mathrm{i}\left(\Omega_{\mathrm{A}} \tau_{\mathrm{A}, 0}+\Omega_{\mathrm{B}} \tau_{\mathrm{B}, 0}\right)}+\alpha \sqrt{1-\alpha^{2}} e^{-\mathrm{i} \theta} f_{(++++)} \\
& +\alpha \sqrt{1-\alpha^{2}} e^{\mathrm{i} \theta} f_{(----)} e^{-\mathrm{i} 2\left(\Omega_{\mathrm{A}} \tau_{\mathrm{A}, 0}+\Omega_{\mathrm{B}} \tau_{\mathrm{B}, 0}\right)}+\left(1-\alpha^{2}\right) f_{(++--)} e^{-\mathrm{i}\left(\Omega_{\mathrm{A}} \tau_{\mathrm{A}, 0}+\Omega_{\mathrm{B}} \tau_{\mathrm{B}, 0}\right)}, \\
& r_{22}=\alpha^{2} f_{(-+-+)}+\alpha \sqrt{1-\alpha^{2}} e^{-\mathrm{i} \theta} f_{(+--+)} e^{\mathrm{i}\left(\Omega_{\mathrm{A}} \tau_{\mathrm{A}, 0}+\Omega_{\mathrm{B}} \tau_{\mathrm{B}, 0}\right)} \\
& +\alpha \sqrt{1-\alpha^{2}} e^{\mathrm{i} \theta} f_{(-++-)} e^{-\mathrm{i}\left(\Omega_{\mathrm{A}} \tau_{\mathrm{A}, 0}+\Omega_{\mathrm{B}} \tau_{\mathrm{B}, 0}\right)}+\left(1-\alpha^{2}\right) f_{(+-+-)}, \\
& r_{23}=\alpha^{2} f_{(+--+)} e^{-\mathrm{i}\left(\Omega_{\mathrm{A}} \tau_{\mathrm{A}, 0}-\Omega_{\mathrm{B}} \tau_{\mathrm{B}, 0}\right)}+\alpha \sqrt{1-\alpha^{2}} e^{-\mathrm{i} \theta} f_{(-+-+)} e^{\mathrm{i} 2 \Omega_{\mathrm{B}} \tau_{\mathrm{B}, 0}} \\
& +\alpha \sqrt{1-\alpha^{2}} e^{\mathrm{i} \theta} f_{(+-+-)} e^{-\mathrm{i} 2 \Omega_{\mathrm{A}} \tau_{\mathrm{A}, 0}}+\left(1-\alpha^{2}\right) f_{(-++-)} e^{-\mathrm{i}\left(\Omega_{\mathrm{A}} \tau_{\mathrm{A}, 0}-\Omega_{\mathrm{B}} \tau_{\mathrm{B}, 0}\right)}, \\
& r_{33}=\alpha^{2} f_{(+-+-)}+\alpha \sqrt{1-\alpha^{2}} e^{-\mathrm{i} \theta} f_{(-++-)} e^{\mathrm{i}\left(\Omega_{\mathrm{A}} \tau_{\mathrm{A}, 0}+\Omega_{\mathrm{B}} \tau_{\mathrm{B}, 0}\right)} \\
& +\alpha \sqrt{1-\alpha^{2}} e^{\mathrm{i} \theta} f_{(+--+)} e^{-\mathrm{i}\left(\Omega_{\mathrm{A}} \tau_{\mathrm{A}, 0}+\Omega_{\mathrm{B}} \tau_{\mathrm{B}, 0}\right)}+\left(1-\alpha^{2}\right) f_{(-+-+)}, \\
& r_{44}=\alpha^{2} f_{(----)}+\alpha \sqrt{1-\alpha^{2}} e^{-\mathrm{i} \theta} f_{(++--)} e^{\mathrm{i}\left(\Omega_{\mathrm{A}} \tau_{\mathrm{A}, 0}+\Omega_{\mathrm{B}} \tau_{\mathrm{B}, 0}\right)} \\
& +\alpha \sqrt{1-\alpha^{2}} e^{\mathrm{i} \theta} f_{(--++)} e^{-\mathrm{i}\left(\Omega_{\mathrm{A}} \tau_{\mathrm{A}, 0}+\Omega_{\mathrm{B}} \tau_{\mathrm{B}, 0}\right)}+\left(1-\alpha^{2}\right) f_{(++++)}, \\
& f_{( \pm \pm \pm \pm)}=\frac{1}{4}\left[1 \pm f_{\mathrm{A}} \pm f_{\mathrm{B}} \cos (2 \Theta)+f_{\mathrm{A}} f_{\mathrm{B}} \cosh \omega\right] \\
& f_{( \pm \pm \mp \mp)}=\mp \frac{1}{4} f_{\mathrm{B}}\left[\mathrm{i} \sin (2 \Theta) \mp f_{\mathrm{A}} \sinh \omega\right] \\
& f_{( \pm \mp \pm \mp)}=\frac{1}{4}\left[1 \mp f_{\mathrm{A}} \pm f_{\mathrm{B}} \cos (2 \Theta)-f_{\mathrm{A}} f_{\mathrm{B}} \cosh \omega\right], \\
& f_{( \pm \mp \mp \pm)}=\mp \frac{1}{4} f_{\mathrm{B}}\left[\mathrm{i} \sin (2 \Theta) \pm f_{\mathrm{A}} \sinh \omega\right] \text {. }
\end{aligned}
$$

These expressions still can be simplified. For simplicity, let us define $\vartheta=\Omega_{\mathrm{A}} \tau_{\mathrm{A}, 0}+\Omega_{\mathrm{B}} \tau_{\mathrm{B}, 0}-\theta$. Let us consider $r_{11}$ as an example. By denoting $f_{( \pm \pm \pm \pm)}=\frac{1}{4}(P \pm Q)$ where $P:=1+f_{\mathrm{A}} f_{\mathrm{B}} \cosh \omega$ and $Q:=f_{\mathrm{A}}+f_{\mathrm{B}} \cos (2 \Theta)$, we get the following.

$$
\begin{aligned}
r_{11} & =\alpha^{2} f_{(++++)}+\alpha \sqrt{1-\alpha^{2}} f_{(--++)} e^{\mathrm{i} \vartheta}+\alpha \sqrt{1-\alpha^{2}} f_{(++--)} e^{-\mathrm{i} \vartheta}+\left(1-\alpha^{2}\right) f_{(----)} \\
& =\frac{1}{4} \alpha^{2}(P+Q)+\frac{1}{4}\left(1-\alpha^{2}\right)(P-Q)+2 \alpha \sqrt{1-\alpha^{2}} \operatorname{Re}\left[f_{(--++)} e^{\mathrm{i} \vartheta}\right] \\
& =\frac{1}{4}\left[P+\left(2 \alpha^{2}-1\right) Q\right]+2 \alpha \sqrt{1-\alpha^{2}} \operatorname{Re}\left[\frac{f_{\mathrm{B}}}{4}\left[\mathrm{i} \sin (2 \Theta)+f_{\mathrm{A}} \sinh \omega\right] e^{\mathrm{i} \vartheta}\right] \\
& =\frac{1}{4}\left[P+\left(2 \alpha^{2}-1\right) Q\right]+\frac{f_{\mathrm{B}}}{2} \alpha \sqrt{1-\alpha^{2}}\left[f_{\mathrm{A}} \sinh \omega \cos \vartheta-\sin (2 \Theta) \sin \vartheta\right] .
\end{aligned}
$$

Via a similar procedure, we obtain the final form $(15 \mathrm{a})-(15 \mathrm{f})$.

Finally, let us derive $f_{j}, \Theta$ and $\omega$ given in (22), 23) and (24). We choose the Gaussian smearing function (21) so that its Fourier transformation is

$$
\tilde{F}(\boldsymbol{k})=\frac{1}{\sqrt{(2 \pi)^{n}}} e^{-|\boldsymbol{k}|^{2} \sigma^{2} / 4} .
$$


Assuming $n=3, f_{j}$ can be evaluated by substituting $\tilde{F}(\boldsymbol{k})$ to $\beta_{j}(\boldsymbol{k})$ (17).

$$
\begin{aligned}
f_{j} & =\exp \left(-\frac{1}{2} \int \mathrm{d}^{n} k\left|\beta_{j}(\boldsymbol{k})\right|^{2}\right) \\
& =\exp \left(-2 \lambda_{j}^{2} \eta_{j}^{2} \int \frac{\mathrm{d}^{n} k}{(2 \pi)^{n} 2|\boldsymbol{k}|} e^{-|\boldsymbol{k}|^{2} \sigma^{2} / 2}\right) \\
& =\exp \left(-2 \lambda_{j}^{2} \eta_{j}^{2} \frac{4 \pi}{(2 \pi)^{3} 2} \int_{0}^{\infty} \mathrm{d}|\boldsymbol{k}||\boldsymbol{k}| e^{-|\boldsymbol{k}|^{2} \sigma^{2} / 2}\right) \\
& =\exp \left(-\frac{\lambda_{j}^{2} \eta_{j}^{2}}{2 \pi^{2} \sigma^{2}}\right)
\end{aligned}
$$

where we have used $\mathrm{d}^{3} k=|\boldsymbol{k}|^{2} \sin \theta \mathrm{d}|\boldsymbol{k}| \mathrm{d} \theta \mathrm{d} \phi$ in the spherical coordinates.

Now let us assume that two detectors are identical, namely, $\lambda:=\lambda_{\mathrm{A}}=\lambda_{\mathrm{B}}, \eta:=\eta_{\mathrm{A}}=\eta_{\mathrm{B}}$, and $\Omega:=\Omega_{\mathrm{A}}=\Omega_{\mathrm{B}}$. $\Theta$ can be computed as

$$
\begin{aligned}
\Theta & =\frac{\mathrm{i}}{4} \int \mathrm{d}^{3} k\left[\beta_{\mathrm{A}}^{*}(\boldsymbol{k}) \beta_{\mathrm{B}}(\boldsymbol{k})-\beta_{\mathrm{A}}(\boldsymbol{k}) \beta_{\mathrm{B}}^{*}(\boldsymbol{k})\right] \\
& =\frac{\mathrm{i}}{4} \frac{4 \lambda^{2} \eta^{2}}{2(2 \pi)^{3}} \int \mathrm{d}^{3} k \frac{e^{-|\boldsymbol{k}|^{2} \sigma^{2} / 2}}{|\boldsymbol{k}|}\left[e^{\mathrm{i}|\boldsymbol{k}|\left(\tau_{\mathrm{B}, 0}-\tau_{\mathrm{A}, 0}\right)} e^{-\mathrm{i} \boldsymbol{k} \cdot\left(\boldsymbol{x}_{\mathrm{B}}-\boldsymbol{x}_{\mathrm{A}}\right)}-\text { c.c. }\right] \\
& =\frac{\mathrm{i} \lambda^{2} \eta^{2}}{2(2 \pi)^{3}} \int_{0}^{\infty} \mathrm{d}|\boldsymbol{k}||\boldsymbol{k}|^{2} \frac{e^{-|\boldsymbol{k}|^{2} \sigma^{2} / 2}}{|\boldsymbol{k}|} \int_{0}^{\pi} \mathrm{d} \theta \sin \theta\left[e^{\mathrm{i}|\boldsymbol{k}| \Delta \tau} e^{-\mathrm{i}|\boldsymbol{k}| L \cos \theta}-\text { c.c. }\right] \int_{0}^{2 \pi} \mathrm{d} \phi \\
& =\frac{\mathrm{i} \lambda^{2} \eta^{2}(2 \pi)}{2(2 \pi)^{3}} \int_{0}^{\infty} \mathrm{d}|\boldsymbol{k}||\boldsymbol{k}| e^{-|\boldsymbol{k}|^{2} \sigma^{2} / 2} \frac{2 \sin (|\boldsymbol{k}| L)}{|\boldsymbol{k}| L}\left(e^{\mathrm{i}|\boldsymbol{k}| \Delta \tau}-e^{-\mathrm{i}|\boldsymbol{k}| \Delta \tau}\right) \\
& =\frac{-2 \lambda^{2} \eta^{2}}{(2 \pi)^{2} L} \int_{0}^{\infty} \mathrm{d}|\boldsymbol{k}| e^{-|\boldsymbol{k}|^{2} \sigma^{2} / 2} \sin (|\boldsymbol{k}| L) \sin (|\boldsymbol{k}| \Delta \tau) \\
& =\frac{\lambda^{2} \eta^{2}}{4 \pi^{2} L \sigma} \sqrt{\frac{\pi}{2}}\left(e^{-(\Delta \tau+L)^{2} / 2 \sigma^{2}}-e^{-(\Delta \tau-L)^{2} / 2 \sigma^{2}}\right) .
\end{aligned}
$$

Also for $\omega$,

$$
\begin{aligned}
\omega & =-\frac{1}{2} \int \mathrm{d}^{3} k\left[\beta_{\mathrm{A}}^{*}(\boldsymbol{k}) \beta_{\mathrm{B}}(\boldsymbol{k})+\beta_{\mathrm{A}}(\boldsymbol{k}) \beta_{\mathrm{B}}^{*}(\boldsymbol{k})\right] \\
& =-\frac{1}{2} \frac{4 \lambda^{2} \eta^{2}}{2(2 \pi)^{3}} \int \mathrm{d}^{3} k \frac{e^{-|\boldsymbol{k}|^{2} \sigma^{2} / 2}}{|\boldsymbol{k}|}\left(e^{\mathrm{i}|\boldsymbol{k}| \Delta \tau} e^{-\mathrm{i} \boldsymbol{k} \cdot \Delta \boldsymbol{x}}+\text { c.c. }\right) \\
& =-\frac{1}{2} \frac{4 \lambda^{2} \eta^{2}}{2(2 \pi)^{3}} \int_{0}^{\infty} \mathrm{d}|\boldsymbol{k}||\boldsymbol{k}|^{2} \frac{e^{-|\boldsymbol{k}|^{2} \sigma^{2} / 2}}{|\boldsymbol{k}|} \int_{0}^{\pi} \mathrm{d} \theta \sin \theta\left(e^{\mathrm{i}|\boldsymbol{k}| \Delta \tau} e^{-\mathrm{i}|\boldsymbol{k}| L \cos \theta}+\text { c.c. }\right) \int_{0}^{2 \pi} \mathrm{d} \phi \\
& =-\frac{1}{2} \frac{4 \lambda^{2} \eta^{2}(2 \pi)}{2(2 \pi)^{3}} \int_{0}^{\infty} \mathrm{d}|\boldsymbol{k}||\boldsymbol{k}| e^{-|\boldsymbol{k}|^{2} \sigma^{2} / 2} \frac{2 \sin (|\boldsymbol{k}| L)}{|\boldsymbol{k}| L} 2 \cos (|\boldsymbol{k}| \Delta \tau) \\
& =-\frac{\lambda^{2} \eta^{2}}{\sqrt{2} \pi^{2} \sigma L}\left[D^{+}\left(\frac{\Delta \tau+L}{\sqrt{2} \sigma}\right)-D^{+}\left(\frac{\Delta \tau-L}{\sqrt{2} \sigma}\right)\right]
\end{aligned}
$$

where $D^{+}(x)$ is the Dawson function.

[1] S. J. Summers and R. Werner, The vacuum violates Bell's inequalities, Phys. Lett. 110A, 257 (1985)

[2] S. J. Summers and R. Werner, Bell's inequalities and quantum field theory. i. general setting, J. Math. Phys. (N.Y.) 28, 2440 (1987)
[3] A. Valentini, Non-local correlations in quantum electrodynamics, Phys. Lett. 153A, 321 (1991).

[4] B. Reznik, Entanglement from the vacuum, Found. Phys. 33, 167 (2003).

[5] B. Reznik, A. Retzker, and J. Silman, Violating Bell's 
inequalities in vacuum, Phys. Rev. A 71, 042104 (2005).

[6] G. V. Steeg and N. C. Menicucci, Entangling power of an expanding universe, Phys. Rev. D 79, 044027 (2009).

[7] A. Pozas-Kerstjens and E. Martín-Martínez, Harvesting correlations from the quantum vacuum, Phys. Rev. D 92, $064042(2015)$

[8] G. Salton, R. B. Mann, and N. C. Menicucci, Acceleration-assisted entanglement harvesting and rangefinding, New J. Phys. 17, 035001 (2015).

[9] E. Martín-Martínez, A. R. H. Smith, and D. R. Terno, Spacetime structure and vacuum entanglement, Phys. Rev. D 93, 044001 (2016)

[10] S. Kukita and Y. Nambu, Harvesting large scale entanglement in de Sitter space with multiple detectors, Entropy 19, 449 (2017).

[11] P. Simidzija and E. Martín-Martínez, Nonperturbative analysis of entanglement harvesting from coherent field states, Phys. Rev. D 96, 065008 (2017).

[12] P. Simidzija, R. H. Jonsson, and E. Martín-Martínez, General no-go theorem for entanglement extraction, Phys. Rev. D 97, 125002 (2018).

[13] L. J. Henderson, R. A. Hennigar, R. B. Mann, A. R. H. Smith, and J. Zhang, Harvesting entanglement from the black hole vacuum, Classical Quantum Gravity 35, 21LT02 (2018)

[14] K. K. Ng, R. B. Mann, and E. Martín-Martínez, UnruhDeWitt detectors and entanglement: The anti-de Sitter space, Phys. Rev. D 98, 125005 (2018).

[15] L. J. Henderson, R. A. Hennigar, R. B. Mann, A. R. Smith, and J. Zhang, Entangling detectors in anti-de Sitter space, J. High Energy Phys. 05 (2019), 178.

[16] W. Cong, E. Tjoa, and R. B. Mann, Entanglement harvesting with moving mirrors, J. High Energy Phys. 06 (2019), 021

[17] E. Tjoa and R. B. Mann, Harvesting correlations in Schwarzschild and collapsing shell spacetimes, J. High Energy Phys. 08 (2020), 155

[18] W. Cong, C. Qian, M. R. Good, and R. B. Mann, Effects of horizons on entanglement harvesting, J. High Energy Phys. 10 (2020), 67.

[19] Q. Xu, S. Ali Ahmad, and A. R. H. Smith, Gravitational waves affect vacuum entanglement, Phys. Rev. D 102, 065019 (2020)

[20] K. Gallock-Yoshimura, E. Tjoa, and R. B. Mann, Harvesting entanglement with detectors freely falling into a black hole, Phys. Rev. D 104, 025001 (2021)

[21] W. G. Unruh, Notes on black-hole evaporation, Phys. Rev. D 14, 870 (1976)

[22] B. S. DeWitt, Quantum gravity: The new synthesis, in General Relativity: An Einstein Centenary Survey, edited by S. W. Hawking and W. Israel (1979) pp. 680745 .

[23] E. Martín-Martínez, M. Montero, and M. del Rey, Wavepacket detection with the unruh-dewitt model, Phys. Rev. D 87, 064038 (2013).

[24] A. M. Alhambra, A. Kempf, and E. Martín-Martínez, Casimir forces on atoms in optical cavities, Phys. Rev. A 89, 033835 (2014).

[25] A. Pozas-Kerstjens and E. Martín-Martínez, Entanglement harvesting from the electromagnetic vacuum with hydrogenlike atoms, Phys. Rev. D 94, 064074 (2016)
[26] P. M. Alsing and G. J. Milburn, Teleportation with a uniformly accelerated partner, Phys. Rev. Lett. 91, 180404 (2003)

[27] I. Fuentes-Schuller and R. B. Mann, Alice falls into a black hole: Entanglement in noninertial frames, Phys. Rev. Lett. 95, 120404 (2005).

[28] Q. Pan and J. Jing, Hawking radiation, entanglement, and teleportation in the background of an asymptotically flat static black hole, Phys. Rev. D 78, 065015 (2008)

[29] A. G. S. Landulfo and G. E. A. Matsas, Sudden death of entanglement and teleportation fidelity loss via the unruh effect, Phys. Rev. A 80, 032315 (2009)

[30] J. Wang, Q. Pan, and J. Jing, Entanglement redistribution in the schwarzschild spacetime, Physics Letters B 692, $202(2010)$

[31] E. Martín-Martínez, L. J. Garay, and J. León, Unveiling quantum entanglement degradation near a schwarzschild black hole, Phys. Rev. D 82, 064006 (2010)

[32] P. M. Alsing, I. Fuentes-Schuller, R. B. Mann, and T. E. Tessier, Entanglement of dirac fields in noninertial frames, Phys. Rev. A 74, 032326 (2006).

[33] E. Martín-Martínez and J. León, Fermionic entanglement that survives a black hole, Phys. Rev. A 80, 042318 (2009)

[34] J. Wang and J. Jing, Quantum decoherence in noninertial frames, Phys. Rev. A 82, 032324 (2010).

[35] M. Montero and E. Martín-Martínez, Entanglement of arbitrary spin fields in noninertial frames, Phys. Rev. A 84, 012337 (2011).

[36] S.-Y. Lin, C.-H. Chou, and B. L. Hu, Disentanglement of two harmonic oscillators in relativistic motion, Phys. Rev. D 78, 125025 (2008)

[37] J. Doukas and B. Carson, Entanglement of two qubits in a relativistic orbit, Phys. Rev. A 81, 062320 (2010)

[38] E. Martín-Martínez and P. Rodriguez-Lopez, Relativistic quantum optics: The relativistic invariance of the light-matter interaction models, Phys. Rev. D 97, 105026 (2018)

[39] E. Martín-Martínez, T. R. Perche, and B. de S. L. Torres, General relativistic quantum optics: Finite-size particle detector models in curved spacetimes, Phys. Rev. D 101, 045017 (2020)

[40] N. Birrell and P. Davies, Quantum Fields in Curved Space, Cambridge Monographs on Mathematical Physics (Cambridge University Press, Cambridge, England, 1984).

[41] W. K. Wootters, Entanglement of formation of an arbitrary state of two qubits, Phys. Rev. Lett. 80, 2245 (1998).

[42] M. Nielsen and I. Chuang, Quantum Computation and Quantum Information, Cambridge Series on Information and the Natural Sciences (Cambridge University Press, Cambridge, England, 2000).

[43] Q. Pan and J. Jing, Degradation of nonmaximal entanglement of scalar and dirac fields in noninertial frames, Phys. Rev. A 77, 024302 (2008).

[44] J. Wang, Q. Pan, S. Chen, and J. Jing, Entanglement of coupled massive scalar field in background of dilaton black hole, Physics Letters B 677, 186 (2009). 